\title{
Energy distribution in nonaxisymmetric magnetic Taylor-Couette flow
}

\author{
M. Gellert ${ }^{1, \star}$, G. Rüdiger ${ }^{1}$, and A. Fournier ${ }^{2}$ \\ 1 Astrophysikalisches Institut Potsdam, An der Sternwarte 16, D-14482 Potsdam, Germany \\ ${ }^{2}$ Laboratoire de Géophysique Interne et Tectonophysique, Université Joseph-Fourier, CNRS, BP 53, 38041 Grenoble \\ cedex 9, France
}

Received 2007, accepted 2007

Published online 2007

Key words magnetohydrodynamics - instabilities

\begin{abstract}
Azimuthal magnetorotational instability is a mechanism that generates nonaxisymmetric field pattern. Nonlinear simulations in an infinite Taylor-Couette system with current-free external field show, that not only the linearly unstable mode $m=1$ appears, but also an inverse cascade transporting energy into the axisymmetric field is possible. By varying the Reynolds number of the flow and the Hartmann number for the magnetic field, we find that the ratio between axisymmetric $(m=0)$ and dominating nonaxisymmetric mode $(m=1)$ can be nearly free chosen. On the surface of the outer cylinder this mode distribution appears similarly, but with weaker axisymmetric fields. We do not find significant differences in the case that a constant current within the flow is added.
\end{abstract}

(C) 2007 WILEY-VCH Verlag GmbH \& Co. KGaA, Weinheim

\section{Introduction}

MHD-instabilities in radiation zones of differentially rotating stars influence the rotation profile of the star due to angular momentum transport (Denissenkov \& Pinsonneault 2007; Yoon, Langer \& Norman 2006). Tayler instability (Tayler 1973) or helical magnetorotational instability (Hollerbach \& Rüdiger 2005) are examples for such instabilities. According to Spruit (1999) the Tayler instability could possibly support a dynamo. In their model the instability should create a significant poloidal field that due to differential rotation is wound up into a toroidal one and thus close the loop for a self-sustained dynamo (Braithwaite \& Spruit 2004; Braithwaite 2006). While this idea sounds promising, there are doubts about its general functionality. Simulations in very similar geometry with $z$-dependent differential rotation did not show a dynamo (Gellert, Rüdiger \& Elstner 2007). Zahn, Brun \& Mathis (2007) claim that such a process is not possible in this simple way. The authors argue that the nonaxisymmetric modes wound up by differential rotation can not contribute to the axisymmetric field, because the azimuthal wavenumber is always $m \neq 0$. As we show in the following, azimuthal magnetorotational instability (AMRI) creates also an axisymmetric component in the nonlinear regime. For a scenario based on AMRI as instability mechanism that might overcome the arguments of the incompatible wavenumber and would make field regeneration at least possible. It does not necessarily mean, however, that it supports a dynamo.

This work consists of two aspects. First we describe the development of nonaxisymmetric modes in the nonlinear

\footnotetext{
^ Corresponding author: mgellert@aip.de
}

regime of AMRI-unstable Taylor-Couette flow. Especially the observed inverse cascade transporting energy into the axisymmetric field and its dependence on the shear is analyzed. As second aspect we point out that weak currents within the flow do not basically influence the instability. Field structure and energy distribution remain almost unchanged.

\section{Equations and numerical treatment}

We use the hydrodynamic Fourier spectral element code described by Fournier et al. (2005) extended by its magnetic field. With this approach we solve the 3D MHD equations

$\partial_{t} \boldsymbol{u}+(\boldsymbol{u} \cdot \nabla) \boldsymbol{u}=-\nabla p+\nabla^{2} \boldsymbol{u}+\frac{\mathrm{Ha}^{2}}{\mathrm{Pm}}(\operatorname{rot} \boldsymbol{B}) \times \boldsymbol{B}$,
$\partial_{t} \boldsymbol{B}=\frac{1}{\mathrm{Pm}} \nabla^{2} \boldsymbol{B}+\operatorname{rot}(\boldsymbol{u} \times \boldsymbol{B})$,
$\operatorname{div} \boldsymbol{u}=0, \quad \operatorname{div} \boldsymbol{B}=0$

for an incompressible medium in cylindrical coordinates

$(R, \phi, z)$. Free parameters are the Hartmann number

$\mathrm{Ha}=B_{\text {in }}^{0} \sqrt{\frac{R_{\text {in }} D}{\mu_{0} \rho \nu \eta}}$

and the magnetic Prandtl number $\operatorname{Pm}=\nu / \eta$. Here $\nu$ is the viscosity of the fluid and $\eta$ its magnetic diffusivity. The Reynolds number is defined as $\operatorname{Re}=\Omega_{\text {in }} R_{\text {in }} D / \nu$ with $D=$ $R_{\text {out }}-R_{\text {in }}$ (unit of length) and the angular velocity of the inner cylinder $\Omega_{\text {in }}$. Unit of velocity is $\nu / D$ and unit of time the viscous time $D^{2} / \nu$.

The solution is expanded in $M$ Fourier modes in the azimuthal direction. This gives rise to a collection of meridional problems, each of which is solved using a Legendre 
spectral element method (see e.g. Deville, Fischer \& Mund 2002). Either $M=8$ or $M=16$ Fourier modes are used, three elements in radial and eighteen elements in axial direction. The polynomial order is varied between $N=8$ and $N=12$. With a semi-implicit approach consisting of second-order backward differentiation formula and third order Adams-Bashforth for the nonlinear forcing terms time stepping is done with second-order accuracy (Fournier et al. 2004).

At the inner and outer wall perfect conducting boundary conditions are applied. In axial direction we use periodic boundary conditions to avoid perturbing effects from solid end caps. The periodicity in $z$ is set to $\Gamma=6 D$.

The initial flow profile is the typical Couette profile

$\Omega(R)=a+\frac{b}{R^{2}}$

with

$a=\frac{\hat{\mu}_{\Omega}-\hat{\eta}^{2}}{1-\hat{\eta}^{2}} \Omega_{\mathrm{in}}, \quad b=\frac{1-\hat{\mu}_{\Omega}}{1-\hat{\eta}^{2}} R^{2} \Omega_{\mathrm{in}}$,

$\hat{\eta}=R_{\text {out }} / R_{\text {in }}=0.5$ and $\hat{\mu}_{\Omega}=\Omega_{\text {out }} / \Omega_{\text {in }}=0.5$. This means the outer cylinder rotates with half of the angular velocity of the inner cylinder in the same direction. After the Rayleigh stability criterion $\partial_{R}\left(R^{2} \Omega\right)^{2}>0$ this configuration is hydrodynamically stable. In addition a toroidal external field $\boldsymbol{B}_{\text {ext }}=\left(0, B^{0}, 0\right)$ is applied. The second component $B^{0}$ has a radial profile similar to the flow:

$B^{0}(R)=a_{B} R+\frac{b_{B}}{R}$.

The external field than can be characterized by the number

$\hat{\mu}_{B}=\frac{B_{\mathrm{out}}^{0}}{B_{\mathrm{in}}^{0}}=\frac{a_{B} R_{\mathrm{out}}+b_{B} R_{\mathrm{out}}^{-1}}{a_{B} R_{\mathrm{in}}+b_{B} R_{\mathrm{in}}^{-1}}$.

The current-free field is given by $\hat{\mu}_{B}=0.5$. Values below lead to a field connected with constant negative current $j_{z}$ within the flow and larger values to a positive current. To trigger the instability the initial magnetic field consists of small random perturbations (with an amplitude of $\left.10^{-6} B_{\mathrm{in}}^{0}\right)$.

\section{Results}

\subsection{Linear instability}

From linear stability theory is known that only the mode $m=1$ is unstable (Rüdiger et al. 2007a), which is reflected in the nonlinear simulations, too. Only $m=1$ starts to grow in the beginning of a simulation where one is in a quasilinear regime. The stability boundaries found with the nonlinear code compared to linear results are shown in Fig. 1 for $\mathrm{Pm}=1, \hat{\mu}_{\Omega}=0.5$ and $\hat{\mu}_{B}=0.5$. Equal to the results in Rüdiger et al. (2007b) we find not only an onset of the instability if the shear (given by the Reynolds number) exceeds a certain threshold for a given magnetic field configuration (constant Ha), but also an upper boundary. Strong shear prevents the instability - from this point of view an instability

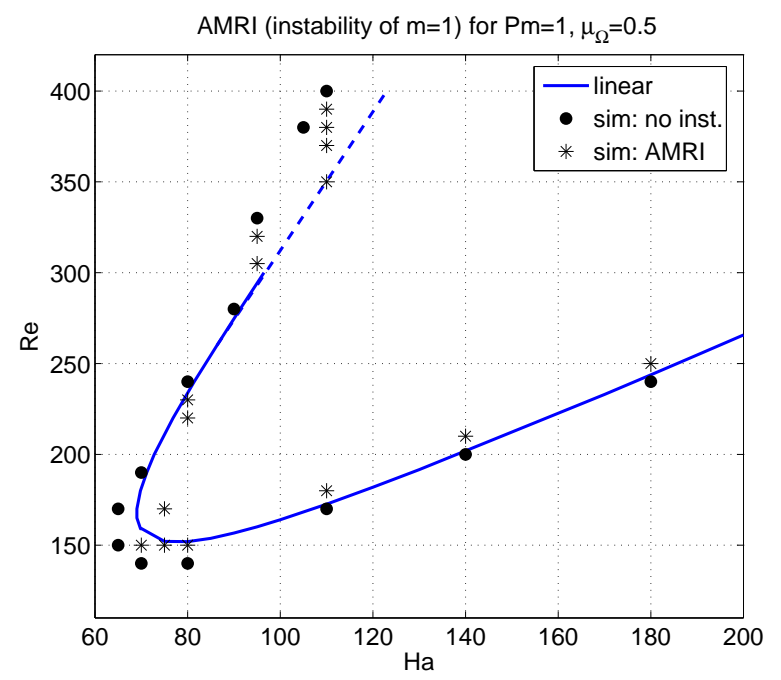

Fig. 1 Stability diagram for $\mathrm{Pm}=1, \hat{\mu}_{\Omega}=0.5$ and $\hat{\mu}_{B}=$ 0.5 (AMRI, current-free external field). The solid line results from linear stability analysis, dots and stars mark results from nonlinear simulations. The dashed line is an assumed continuation of the solid line, there were no linear data available.

of the magnetic field. Both, the linear results and nonlinear simulations, agree very well on the lower boundary. At the upper boundary for Re $>300$ the simulations exhibit a shift of the stability boundary to slightly higher values of Re. But the qualitative picture is the same.

Following a line with constant Re, one can argue that weak but not to small magnetic fields lead to an instability of the flow. For higher Re also the magnetic field has to exceed a higher threshold to sustain the nonaxisymmetric mode generation. Insofar both descriptions, unstable flow due to a magnetic field and magnetic field instability due to shear, are equivalent.

\subsection{Nonlinear simulations}

\subsubsection{Current-free external field}

After the onset of the instability, first exclusively the $m=1$ mode grows consistent with the linear theory. If the $m=1$ mode reaches roughly $30 \%$ the amplitude of the final state, also both neighboring modes $m=0,2$ start to grow. Slightly later successively higher and higher modes follow. The energy drops by a factor of ten for each $m \rightarrow m+1$. Modes $m>3$ carry less than $10^{-3}$ of the total energy and are not plotted in Fig. 4. A time series of the total magnetic energy

$E(m)=\frac{1}{2 V} \int_{V} \boldsymbol{B}^{2}(m) \mathrm{d} V$

for each of the significant modes is shown in Fig. 2, the flow and magnetic field pattern is shown in Fig. 3. Compared to the strength of the external field, the magnetic field due to the AMRI is weaker. The maximum size is around 0.3 $B_{\mathrm{i} n}^{0}$ and the energy below $10 \%$. The saturated state shows no regular time-dependence, the field pattern rotates with a 


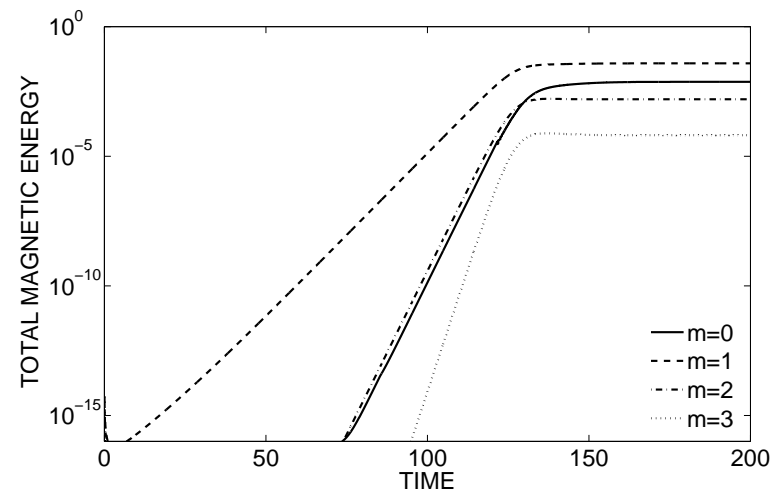

Fig. 2 Time series of the total magnetic energy for Fourier modes $0 \leq m \leq 3$ for $\operatorname{Re}=200$. Linearly unstable is only $m=1$. Time is scaled in periods of rotation.
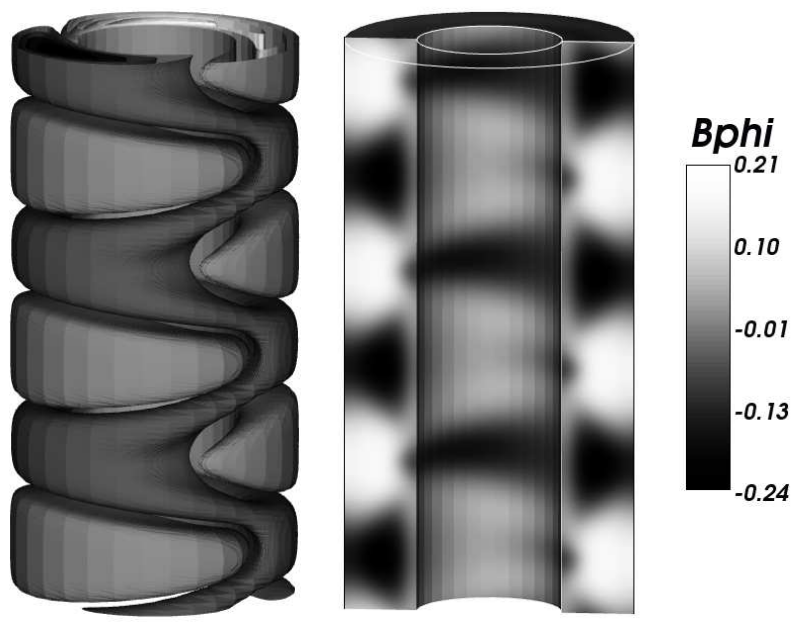

Fig. 3 On the left an isosurface plot of $B_{R}\left( \pm 0.7\left|B_{R}\right|_{\max }=\right.$ $0.09 B_{\mathrm{in}}^{0}$ ) for $\mathrm{Re}=200, \mathrm{Ha}=110$ at $t=300$ is shown, on the right the magnetic field component $B_{\phi}$ in the $R$-z-plane also in units of $B_{\text {in }}^{0}$.

small drift relative to the system rotation (as already pointed out by the linear analysis).

Interesting is the fact that in the nonlinear simulations the modes $m>1$ appear only very weak. The energy of $m=5$ is already by a factor of $10^{5}$ less than the energy of the mode $m=1$. On the other hand also a significant large mode $m=0$ develops. This means energy transport happens not only to higher modes or smaller scales but also in the opposite direction ${ }^{1}$.

By varying the shear strength, the amount of energy in the produced axisymmetric mode can be nearly free chosen. Near the stability boundary (e.g. Re $=175$ and $\mathrm{Re}=390$ for $\mathrm{Ha}=110$, see Fig. 1) the nonaxisymmetric mode $m=1$ dominates the energy spectrum, in between at $\operatorname{Re}=280 m=0$ becomes of the same size.

${ }^{1}$ Regarding a dynamo based on the instability, Zahn et al. (2006) point out that the generation of an axisymmetric field from a nonaxisymmetric one is difficult. If the instability itself also creates an axisymmetric mode the generation of an appropriate poloidal axisymmetric field might be easier to achieve.
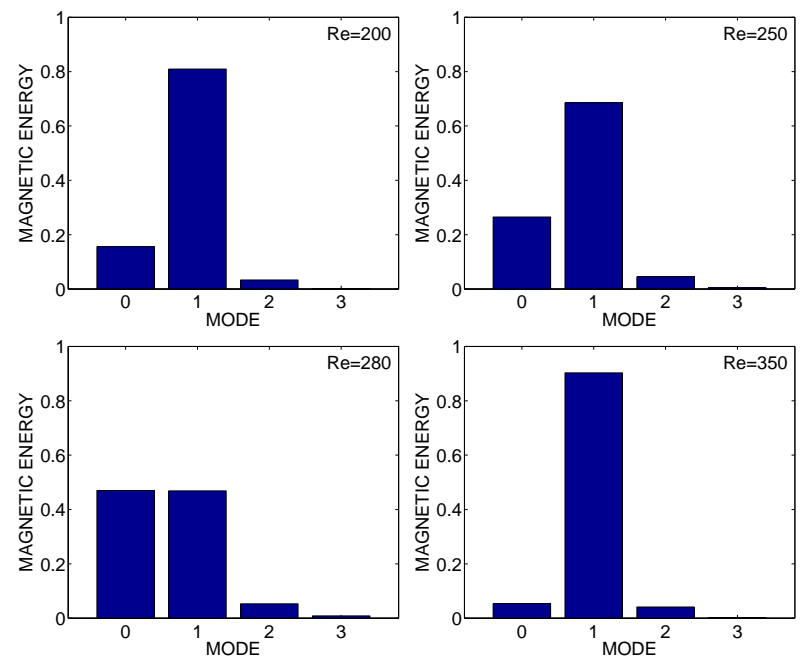

Fig. 4 Total magnetic energy distribution in Fourier modes $m$ with growing shear for $\mathrm{Ha}=110$. The instability is observable between $\operatorname{Re}=175$ and $\operatorname{Re}=390$.

This seems to be a general behavior and is not only the case for $\hat{\mu}_{\Omega}=0.5$. Also for steeper rotation laws, e.g. the quasi-keplerian $\left(\hat{\mu}_{\Omega}=0.3535\right)$, this kind of energy transport into the axisymmetric mode is observable in the simulations. The amount of energy in the axisymmetric mode is always of the order of $50 \%$ of the whole energy or less. This agrees with magnetic fields observed in Ap stars. According to this observations slow rotating Ap stars with strong fields often possess a nonaxisymmetic field. And the axiand nonaxisymmetric modes are of the same size (Landstreet \& Mathys 2000; Bychkov, Bychkova \& Madej 2005). Regarding these observations more interesting than the volume averaged energy is the behavior of the magnetic field that penetrates the surface and would be visible from outside. This is the poloidal part of the field, which consists due to the boundary conditions only of the $z$-component. Looking on the poloidal field, the situation is similar to the global field distribution. The dominating mode is yet the $m=1$ mode. Different is the behavior of the produced axisymmetric mode. It is nearly not visible on the surface. And opposite to the global behavior it becomes even less with Reynolds numbers in the middle of the unstable parameter space, where the energy of the $m=0$ mode is maximal in the volume-averaged quantity. This is illustrated in Fig. 5, where the power spectral density $\left|\tilde{B}_{z}(m)\right|^{2}$ at $R=R_{\text {out }}$ and $z=1 / 3 \Gamma$ is plotted for different Reynolds numbers. The created axisymmetric component contributes mainly to the $B_{\phi}$-component, the original source of the instability. Due to the perfect conducting boundary conditions, which are quite different to the vacuum environment for real stars, the surface field is not directly comparable with the observations. 


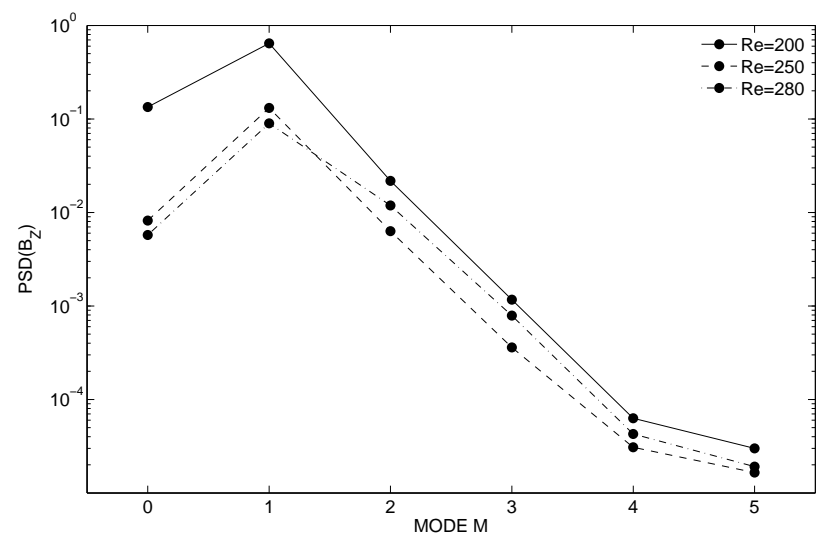

Fig. 5 Power spectral density for $B_{z}$ on the cylinder surface for several $m$ normalized by the total power on the surface. The distribution is similar to the volume-averaged values, except the $m=0$ mode occurs to be smaller in $B_{z}$.

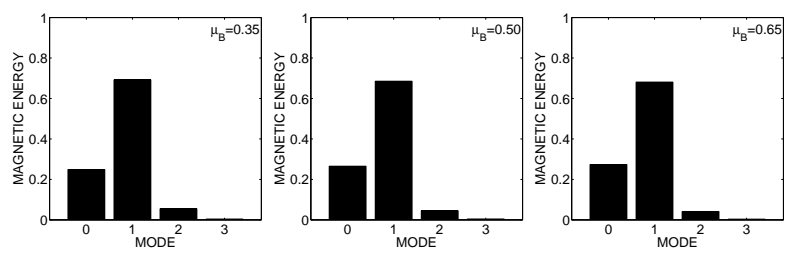

Fig. 6 Influence of a positive or negative current on the energy distribution $\left(\hat{\mu}_{B}=0.35,0.5,0.65\right.$ from left to right $)$ is not visible. The axisymmetric mode carries $E_{0}=0.249,0.265,0.272 \%$ of the total energy, the $m=1$ mode $E_{1}=0.690,0.685,0.682 \%$ for $\hat{\mu}_{B}=0.35,0.5,0.65$. Histograms are normalized with total magnetic energy.

\subsubsection{External field connected with a current}

With the profile of the external field as defined in Eq. (7), an associated current is always constant. It is positive if $\hat{\mu}_{B}>0.5$ and negative if $\hat{\mu}_{B}<0.5$. To test the influence of its current on the appearance of the instability and the resulting energy distribution, we alter the external field profile to $\hat{\mu}_{B}=0.35$ and $\hat{\mu}_{B}=0.65$. These modifications of the external field are small enough not to change the profile qualitatively. The simulations with the modified external fields do not show a basic influence in the energy distribution from a current. The ratio between magnetic energy of modes $m=0$ and $m=1$ has values of $E_{0} / E_{1}=0.37$ for $\hat{\mu}_{B}=0.35, E_{0} / E_{1}=0.39$ for $\hat{\mu}_{B}=0.50$ and $E_{0} / E_{1}=0.40$ for $\hat{\mu}_{B}=0.65$. With increasing $\hat{\mu}_{B}$ also the energy of the external field increases and is responsible for the slightly increasing energy ratio. The instability is not strongly effected by low currents. For larger currents, or larger Hartmann numbers, the influence of the Tayler instability grows and the Reynolds number dependence of the onset of the instability is lost. Tayler instability already works with low or even without shear.

\section{Conclusion}

In an infinite Taylor-Couette system we have shown, that nonlinear simulations of AMRI lead to an unstable nonaxisymmetric mode $m=1$ of the magnetic field if a certain strength of the external magnetic field is reached. Furthermore the instability is suppressed if the external field becomes too strong (for given Reynolds number). Equivalent is the point of view of an instability of the magnetic field due to shear. Here also a certain strength of the shear or value of the Reynolds number has to be reached to trigger the instability of the $m=1$ mode. And if the shear exceeds a certain value, the instability is not observable anymore.

The simulations reveal in the saturated state a distribution of magnetic energy over several modes, not only the $m=1$ is excited. However, the energy transport works in both directions, not only in the small-scale direction to larger $m$, but also to lower $m$. A significant amount of energy is shifted inversely into the axisymmetric mode $(m=0)$. The shear strength is responsible for the ratio between magnetic energy in axisymmetric and nonaxisymmetric mode $m=1$. Near the stability boundaries, the nonaxisymmetric mode carries nearly all energy. In the central region of the unstable parameter space the energy ratio between modes $m=0$ and $m=1$ is roughly one.

Adding a small positive or negative current within the flow does not affect the instability, the field pattern and final energy distribution do not change compared to the currentfree case. A small deviation from the profile $B^{0} \propto R^{-1}$ does not visibly influence the occurrence of the instability.

\section{References}

Braithwaite, J.: 2006, A\&A 449, 451

Braithwaite, J., Spruit, H.C.: 2004, Nature 43, 819

Bychkov, V.D., Bychkova, L.V., Madej, J.: 2005, A\&A 430, 1143

Denissenkov, P.A., Pinsonneault, M.: 2007, ApJ 655, 1157

Deville, M.O., Fischer, P.F., Mund, E.H.: 2002, High Order Methods for Incompressible Fluid Flow, Cambridge University Press

Fournier, A., Bunge, H.-P., Hollerbach, R., Vilotte, J.-P.: 2004, GeoJI 156, 682

Fournier, A., Bunge, H.-P., Hollerbach, R., Vilotte, J.-P.: 2005, JCoPh 204, 462

Gellert, M., Rüdiger, G., Elstner, G.: 2007, A\&A, submitted

Hollerbach, R., Rüdiger, G.: 2005, PhRvL 95, 124501

Landstreet, J.D., Mathys, G.: 2000, A\&A 359, 213

Rüdiger, G., Hollerbach, R., Schultz, M., Elstner, D.: 2007a, MNRAS 377, 1481

Rüdiger, G., Hollerbach, R., Gellert, M., Schultz, M.: 2007b, AN 328, this issue

Spruit, H.C.: 1999, A\&A 349, 189

Tayler, R.J. : 1973, MNRAS 161, 365

Yoon, S.-C., Langer, N., Norman, C.: 2006, A\&A 460, 199

Zahn, J.P., Brun, A.S., Mathis, S.: 2007, A\&A, in press, arXiv: 0707.3287 